\title{
Spontaneous Rupture of Pseudoaneurysm of Gastroduodenal Artery Complicating Pancreatitis: MDCT Diagnosis
}

\author{
${ }^{1}$ P L Sah, ${ }^{1}$ R K Rauniyar, ${ }^{2}$ R K Gupta, ${ }^{1}$ K Dhungel, ${ }^{1}$ K Ahmad, ${ }^{1}$ M K Gupta
}

${ }^{1}$ Department of Radio-diagnosis, ${ }^{2}$ Department of Surgery, B. P. Koirala Institute of Health Sciences (BPKIHS), Dharan, Nepal

\begin{abstract}
Pseudoaneurysm of gastroduodenal artery is least common of all the splanchnic artery aneurysms. It occurs most commonly due to pancreatitis. Spontaneous rupture of the pseudoaneurysm has a high mortality rate. Pseudoaneurysm was traditionally diagnosed by conventional angiography. We report an interesting case of spontaneously ruptured pseudoaneurysm of gastroduodenal artery complicating pancreatitis diagnosed by multidetector computed tomography (MDCT) in a 50 years old man who presented with sudden onset of epigastric pain for 2 days. Contrast enhanced MDCT revealed a large hematoma surrounding pseudoaneurysm of the gastroduodenal artery. The patient was referred for embolization. Since rupture rate and mortality are high, prompt diagnosis of the ruptured pseudoaneurysm helps prevent or decrease mortality and morbidity.
\end{abstract}

Keywords: MDCT, gastroduodenal artery pseudoaneurysm, pancreatitis, spontaneous rupture

\section{Introduction}

Gastroduodenal artery aneurysms (GDA) are the least common of all splanchnic artery aneurysms. Pseudoaneurysm formation is a known vascular complication of pancreatitis. The pseudoaneurysm has been reported to occur in $3.5-10 \%$ of patients with pancreatitis. The arteries involved include, in order of frequency, the splenic (40\%), gastroduodenal $(30 \%)$, pancreaticoduodenal (20\%), gastric $(5 \%)$, hepatic $(2 \%)$, others (superior mesenteric, jejunal, ileocaecal, aorta) $(1-3 \%) .^{1}$ Spontaneous rupture of gastroduodenal artery pseudoaneurysm is associated with high rate of morbidity and mortality. We report a case of spontaneously ruptured pseudoaneurysm of the gastroduodenal artery complicating pancreatitis diagnosed by MDCT.

Correspondence to: Dr. Panna Lal Sah, Associate Professor, Dept. of Radiodiagnosis \& Imaging, BPKIHS, Dharan, Nepal

Email: pannalalsah76@yahoo.com

\section{Case report}

A 50 year old gentle man, who was chronic alcoholic presented to our emergency department with history of sudden onset epigastric pain for 2 days. There was no past history of hospital admission. On examination, patient was slightly pale. However, his vitals were stable. Blood tests were within normal limits except for hemoglobin level of $9 \mathrm{gm} \%$ and serum amylase of 700 SU. Contrast enhanced MDCT scan revealed a non-enhancing mass in the pancreatic head, with a small round contrast filled structure just medial to the gastroduodenal artery. There was also soft tissue stranding in the peripancreatic region and mild left pleural effusion. Based on the radiological findings, combined with the clinical history of the patient, the diagnosis of ruptured pseudo-aneurysm of the gastroduodenal artery caused by 

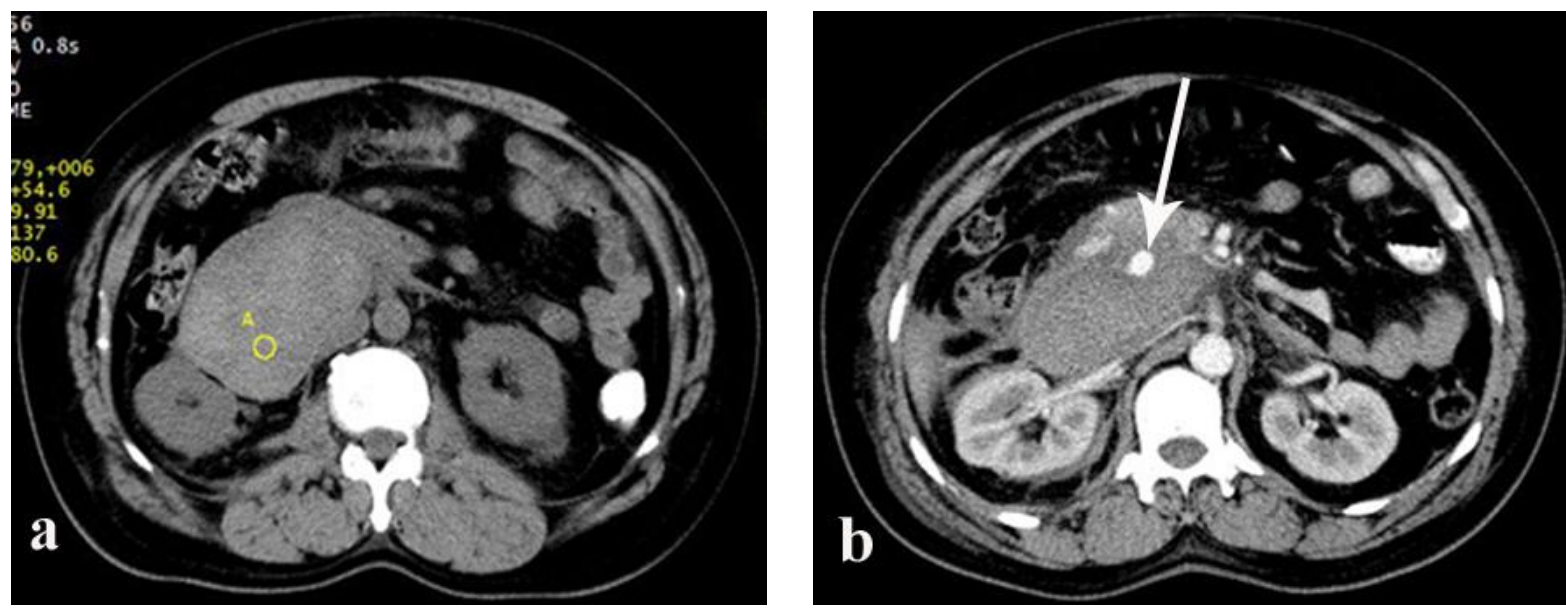

Figure 1 (a, b): Non Contrast CT scan, axial section (a) shows homogeneous high density mass (54 HU) and axial contrast enhanced CT image (b) shows a well defined contrast filled round structure (arrow) within the surrounding hematoma with enhancement similar to aorta s/o aneurysm.
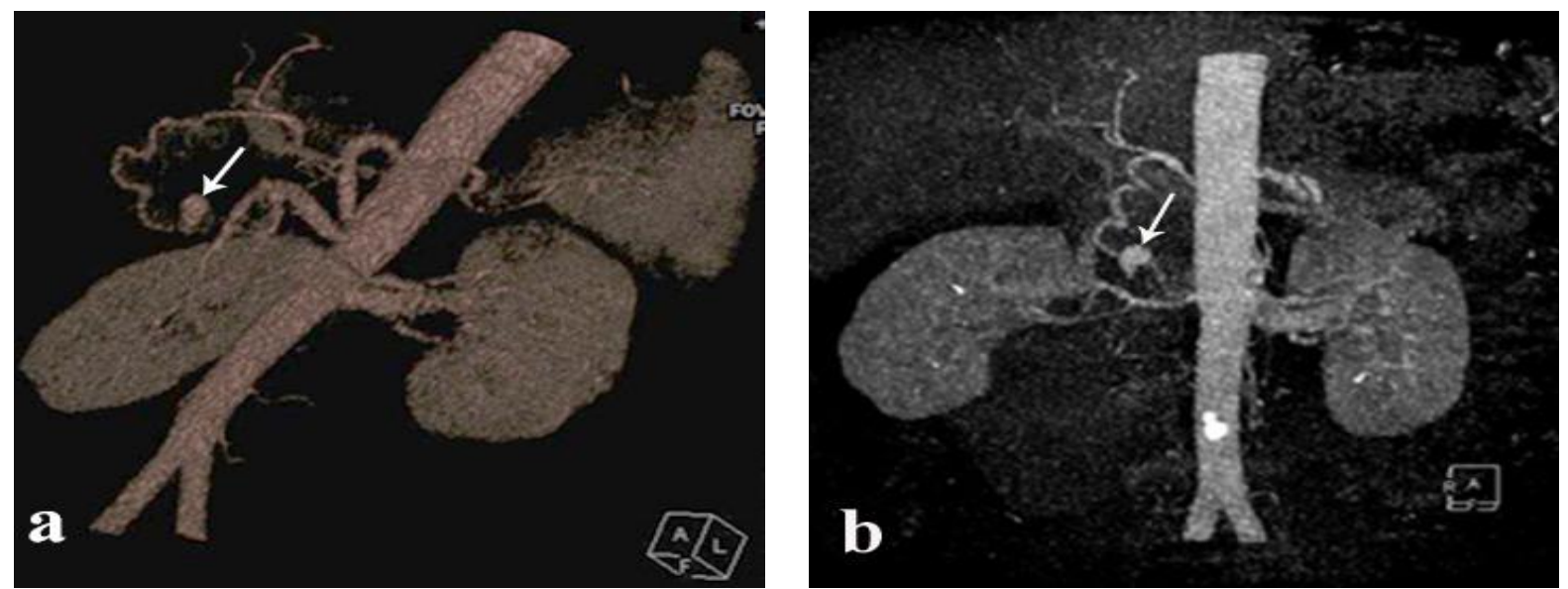

Figure 2 (a, b): (a) MIP and (b) VR image depicts the pseudoaneurysm very well (arrows).

pancreatitis and leading to hematoma was made. The patient was referred for embolization of the pseudoaneurysm.

\section{Discussion}

GDA represent only $1.5 \%$ of all splanchnic artery aneurysms. The majority of them are false or pseudoaneurysms. The first case report of gastroduodenal artery aneurysm was done by Abrams RM way back in $1969 .{ }^{2}$ The most common cause is periarterial inflammation, which usually occurs as a consequence of pancreatitis with vascular necrosis or vessel erosion by an adjacent pseudocyst. These aneurysms are among the most hazardous of all spanchnic artery aneurysms.

They can be asymptomatic, or present with gastrointestinal, intraperitoneal or retroperitoneal bleeding. Rupture of gastroduodenal aneurysms occur in $75 \%$ of inflammatory lesions. ${ }^{3}$ Haemorrhage has been associated with mortality rates exceeding $50 \%$.

The survival of the patient with pancreatitis and ruptured pseudoaneurysm depends on early suspicion and prompt diagnosis. They may be demonstrated by ultrasonography, 
MDCT, magnetic resonance imaging or arteriography. MDCT is a useful non invasive investigative tool for detecting pseudoaneurysm and other vascular complications in pancreatitis. ${ }^{4}$ Angiography has been traditionally considered the standard for diagnosing pancreatic pseudoaneurysm. It has both diagnostic and therapeutic role.

Transarterial embolization as a definite treatment for bleeding pseudoaneurysm has been reported with success rate as high as 80 to $100 \%$. Surgery is necessary in patients with bleeding vessels that are impossible to embolize or for whom angiographic attempts at hemostasis has failed. As risk for rupture is unrelated to size, any gastroduodenal artery aneurysm should be considered for definitive treatment once the diagnosis has been made.

In summary, early suspicion and prompt diagnosis of this complication of pancreatitis would reduce morbidity and mortality of patients with pancreatitis. Physician might face diagnostic challenge in patient who present with pain abdomen with pallor for the first time. Ruptured pseudoaneurysm should be kept in differential diagnosis of patient at risk for pancreatitis and presenting with pallor. MDCT is a useful noninvasive modality for its diagnosis.

\section{References}

1. Mallick IH, Winslet MC. Vascular complications of pancreatitis. JOP. 2004 Sep 10;5(5):328-37.

2. Abrams RM, Kulkarni AV, Beranbaum ER, Santos JS. Aneurysm of the gastro-duodenal artery. $\mathrm{Br} \mathrm{J}$ Radiol. 1969 May;42(497):384-5.

3. Stanley JC. Mesenteric arterial occlusive and aneurysmal disease. Cardiol Clin. 2002 Nov;20(4):61122, vii. Review.

4. Urban BA, Curry CA, Fishman EK. Complications of acute pancreatitis: helical CT evaluation. Emerg Radiol 1999;6:113-20. 